\title{
Intraoperative radiotherapy for breast cancer treatment efficiently targets the tumor bed preventing breast adipose stromal cell outgrowth
}

\author{
Stefanie Uhlig ${ }^{1,2} \cdot$ Anne Wuhrer ${ }^{3} \cdot$ Sebastian Berlit ${ }^{3} \cdot$ Benjamin Tuschy $^{3}$ Marc Sütterlin Maren Bieback $^{1,2}$ (D)
}

Received: 30 October 2019 / Accepted: 16 January 2020 / Published online: 6 February 2020

(C) The Author(s) 2020

\begin{abstract}
Objectives Mesenchymal stromal cells (MSC) in bone marrow have been shown to be radioresistant, which is related to pronounced DNA repair mechanisms. Intraoperative radiotherapy (IORT) during breast-conserving surgery for early breast cancer is an innovative technique applying low energy x-ray to the tumor bed immediately after removal of the tumor. IORT is considered to reduce the risk of local tumor recurrence by directly targeting cells of the tumor bed and altering the local microenvironment. Aim of this study was to investigate whether IORT affects the outgrowth potential of breast adipose tissue-derived MSC (bASC) as part of the tumor bed.

Materials and methods After surgical tumor resection, biopsies of the tumor bed were taken before (pre IORT) and after IORT (post IORT) and processed applying well-established protocols for ASC isolation and characterization.

Results In all, $95 \%$ of pre IORT tumor bed samples yielded persistently outgrowing bASC with typical ASC characteristics: fibroblastoid morphology, proliferation, adipogenic and osteogenic differentiation and ASC surface marker expression. However, none of the post IORT samples yielded persistent outgrowth of bASC.

Conclusions After breast-conserving surgery, approximately $90 \%$ of local recurrences emerge in close proximity to the initial tumor bed, potentially reflecting a significant contribution of the tumor bed to relapse. Our data show that IORT, besides the proven effect on breast cancer cells, efficiently modifies the tumor environment by having an impact on tumor bed bASC. This effect on tumor bed stromal cells might contribute to reduce the risk of tumor relapse and metastases.
\end{abstract}

Keywords Intraoperative radiotherapy $\cdot$ Mesenchymal stromal cells $\cdot$ Adipose stromal cells $\cdot$ Tumor bed $\cdot$ Breast cancer

The authors S. Uhlig and A. Wuhrer contributed equally to the manuscript.

Parts of this work have been presented as poster and oral abstracts and are published as conference abstracts: P09-17 Uhlig S, Wuhrer A, Sütterlin M, Tuschy B, Berlit S, Bieback K. Intraoperative radiotherapy for breast cancer treatment efficiently prevents breast adipose tissue-derived mesenchymal stromal cells outgrowth. Transfus Med Hemother 2017;44(suppl 1):1-94 Abstract No. P024: Intraoperative radiotherapy for breast cancer treatment efficiently prevents breast adipose tissue-derived mesenchymal stromal cells outgrowth. Stefanie Uhlig, Anne Wuhrer, Marc Sütterlin, Benjamin Tuschy, Sebastian Berlit, Karen Bieback. GermanStemCellNetwork 5th Annual Conference 2017. VS-6-4Intraoperative radiotherapy of breast cancer-influence on mesenchymal stromal cells and the tumor bed's micromilieu. Anne Wuhrer, Stefanie Uhlig, Benjamin Tuschy, Sebastian Berlit, Marc Sütterlin, Karen Bieback. Transfus Med Hemother 2019;46(suppl 1):1-87.
Electronic supplementary material The online version of this article (https://doi.org/10.1007/s00066-020-01586-z) contains supplementary material, which is available to authorized users.

Prof. Dr. rer. nat. Karen Bieback

karen.bieback@medma.uni-heidelberg.de

1 Institute of Transfusion Medicine and Immunology, Medical Faculty Mannheim, German Red Cross Blood Donor Services, Heidelberg University, Friedrich-Ebert Str. 107, 68167 Mannheim, Germany

2 FlowCore Mannheim, Medical Faculty Mannheim, Heidelberg University, Ludolf-Krehl Str. 13-17, 68167 Mannheim, Germany

3 Department of Obstetrics and Gynecology, University Medical Center Mannheim, Heidelberg University, Theodor-Kutzer-Ufer 1-3, 68167 Mannheim, Germany 


\begin{tabular}{ll}
\multicolumn{2}{l}{ Abbreviations } \\
ASC & Adipose stromal cells \\
bASC & Breast-derived adipose stromal cells \\
DMEM & Dulbecco's Modified Eagle Medium \\
DMSO & Dimethyl sulfoxide \\
EDTA & Ethylenediaminetetraacetic acid \\
EMT & Epithelial mesenchymal transition \\
FBS & Fetal bovine serum \\
Gy & Gray \\
IORT & Intraoperative radiotherapy \\
ISCT & International Society for Cell \& Gene Therapy \\
min & Minutes \\
ml & Milliliters \\
MSC & Mesenchymal stromal cells \\
PBS & Phosphate-buffered saline \\
RT & Room temperature \\
SNB & Sentinel node biopsy
\end{tabular}

\section{Introduction}

Breast cancer is the most frequent malignant tumor in women. Beside surgical, chemotherapeutic and receptortargeted therapy, local radiotherapy completes the mainstays of treatment. While postoperative whole breast radiotherapy remains standard in patients undergoing breastconserving surgery, intraoperative radiotherapy (IORT) is increasingly implemented in clinical routine. This riskadapted concept uses low energy x-rays applied during surgery directly after excision of the tumor [1]. The idea of using local radiotherapy in general is to eliminate potentially remaining tumor cells in the tumor bed after surgery, considered as a major source for relapse [2]. With irradiation taking place in the wound cavity directly after having removed the tumor, the risk of local or temporal miss is hypothetically nonexistent.

As supported by the seed and soil theory, the wound healing process after surgery is likely to provide favorable growth conditions not only for the healthy tissue, but also for residual tumor clusters [3]. Subsequently, a modification of the tumor bed stroma and its micromilieu as potentially provoked by IORT could result in a reduction of the risk of local recurrence. Furthermore, the fact that local control is correlated with an improvement in overall survival in an oncological disease with early metastatic spread implies that systemic progress might be substantially affected by mechanisms in the tumor bed [4]. IORT could provide a saturation of the DNA repair system eventually leading to increased genomic instability and thus inactivation of tumor cells [2]. Furthermore, immediate irradiation after excision of the primary tumor could prevent the proliferation and division of residual malignant cells during wound healing [2]. In the scope of breast cancer therapy, the impact of
IORT on the tumor bed stroma under in vivo conditions is scarcely investigated, mainly focusing on the wound fluid and not on the cellular part of the tumor bed tissue $[5,6]$.

Mesenchymal stromal cells (MSC), as a potential part of the tumor bed stroma, comprise a heterogeneous population of multipotent stem/stromal cells that can be isolated from a variety of different tissues including adipose tissue (adipose stromal cells, ASCs) [7]. Due to their regenerative potential, MSCs are considered as promising candidates for diverse clinical applications in cell and gene therapy. In this respect, the fate of MSCs under the influence of ionizing radiation became of particular interest. MSCs have been ascribed with evincing radioprotective and regenerative features in tissues exposed to ionizing radiation, even in patients $[8,9]$. Yet, what presents itself as a benefit on the one hand could be considered as a drawback for the oncological outcome, since these protective effects could not only support normal tissue but also tumor cells treated with radiotherapy [9].

In allogeneic bone marrow transplant setting, stromal cells remain host-derived irrespective of the condition regime intensity [10]. This suggests relative radio- and chemoresistance. In fact, ex vivo cultured MSC/ASC are resistant to radiation withstanding even high radiation doses [11].

The aim of this work was to analyze whether IORT affects the outgrowth potential of bASC, indicative for an effect on the tumor bed stroma. Biopsies of breast adipose tissue were harvested in patients with IORT before and after IORT and in control patients without IORT. Outgrowing cells were characterized against MSC criteria.

\section{Materials and methods}

\section{Patients and intraoperative radiotherapy}

A total of 20 breast cancer patients undergoing breast-conserving surgery with (study collective) and 21 without (control collective) IORT were recruited after written informed consent was obtained. All procedures performed in studies involving human participants were in accordance with the ethical standards of the institutional and/or national research committee (No. 2013-589N-MA, Mannheim Ethics committee II) and with the 1964 Helsinki declaration and its later amendments or comparable ethical standards. In women of the study collective, tumor bed biopsies were taken before (pre) and after (post) IORT.

IORT was performed according to the TARGIT-A protocol [4]: The Intrabeam ${ }^{\circledR}$ system (Carl-Zeiss Meditec AG, Oberkochen, Germany) was used for intraoperative irradiation $(50 \mathrm{KeV}$ x-ray). After excision of the tumor and pathoanatomical confirmation of free margins via frozen 
section, the spherical Intrabeam applicator was adjusted in the wound cavity. Irradiation was accomplished with a dosage of $20 \mathrm{~Gy}$. In patients undergoing breast-conserving surgery without IORT, biopsies of the tumor bed were taken pre and post sentinel node biopsy (SNB) to ensure a comparable time interval of approximately $30 \mathrm{~min}$ between both biopsies. Biopsies were taken using conventional scissors. Of course, patients of the control collective also underwent irradiation but in contrast to the study collective solely 3 to 4 weeks after surgery via conventional whole breast radiotherapy.

Histological subtypes and molecular phenotype of were comparable for IORT and controls (not shown).

\section{ASC isolation and characterization}

Twenty-four hours after biopsy, breast ASC (bASC) were isolated using an established method [7]. Briefly, tissue was weighed and then cut into small pieces. The tissue pieces were digested in $10 \mathrm{ml} 0.075 \%$ collagenase I (Sigma Aldrich) in prewarmed DMEM (Pan Biotech) for $30-45 \mathrm{~min}$ at $37^{\circ} \mathrm{C}$. $20 \mathrm{ml}$ of prewarmed DMEM were added to dilute collagenase and then the cell suspension filtered through a $100 \mu \mathrm{m}$ cell strainer (Falcon). The cell suspension was then centrifuged $(1200 \times \mathrm{g}, 10 \mathrm{~min}, \mathrm{RT})$ and then optionally treated with erythrocyte lysis buffer $(1 \times$, $10 \mathrm{~min}$, centrifugation $1200 \times \mathrm{g}, 10 \mathrm{~min}, \mathrm{RT})$. The pellet was resuspended and counted. Cells were plated in a T25 flask (Nunc easy Flask) in DMEM low glucose, penicillin/ streptomycin (Sigma Aldrich), L-glutamine (Gibco) and $10 \%$ human AB-serum (German Red Cross Blood Donor Service). Medium was changed bi-weekly until bASC reached a subconfluent stage (approximately 75\%). Cells were then split using trypsin/EDTA. Cells were counted and aliquots cryopreserved in fetal bovine serum (FBS)/10\% DMSO (dimethyl sulfoxide, WAK-Chemie). Remaining cells were seeded as passage 1 (p1) cells at 200 cells $/ \mathrm{cm}^{2}$. ASC growth rate was monitored recording cell number at every passage by calculating cell doublings (CD) and doubling time (DT):

$$
\begin{aligned}
& \text { cell doublings }(C D) \\
& =\frac{\log 10(F c n)-\log 10(\text { Icn })}{\log 10(2)} \text { doublingtime }(D T) \\
& =\frac{\text { Culture duration }(h)}{C D}
\end{aligned}
$$

where Fcn is final cell number and Icn the initial cell number.

To define multipotent MSCs, the International Society for Cell and Gene Therapy has suggested a characteristic combination of functional properties and the specific surface marker phenotype. The hallmarks include plas- tic adherence, trilineage differentiation capability into adipocytes, chondrocytes and osteoblasts as well as the characteristic surface molecular marker expression, defined by the absence of hematopoietic markers and the presence of CD73, CD90, and CD105 (14,15). After p1, cells were subjected to differentiation assays. Immunophenotyping was performed after $\mathrm{p} 2$. Growth curves were calculated in $\mathrm{p} 1$ and $\mathrm{p} 2$.

\section{Differentiation assays}

Osteogenic and adipogenic differentiation was performed as described previously [7] using osteogenic and adipogenic induction/maintenance medium (Lonza). Briefly, after 21 days of induction, cells were stained with Oil Red $\mathrm{O}$ and von Kossa stain as described.

\section{Immunophenotyping}

Multicolor immunophenotyping was also performed as described before [7]. Briefly, cells were trypsinized using trypsin/EDTA (Pan Biotech) and adjusted to $1 \times 10^{5}$ cells/ tube. FcR blocking reagent (Miltenyi Biotech) was added and incubated for $10 \mathrm{~min}$ before adding the titrated volume of antibody (see table S1). Unstained cells served as negative control. Cells were incubated $20 \mathrm{~min}$, followed by two washes with PBS. Finally, Sytox Blue was added to exclude dead cells and cells were analyzed using a FACS Canto II analyzer running FACS Diva software (Becton Dickinson).

\section{Statistical analysis}

Analysis was performed using GraphPad Prism 7. Significance testing was done using two-way analysis of variance (ANOVA) or $t$-test, as applicable.

\section{Results}

\section{Isolation success rate, phenotype}

MSCs were isolated using collagenase digestion from breast tissue biopsies pre and post IORT (controls: pre and post sentinel lymph node resection). The sample weight was

Table 1 Isolation success in percent

\begin{tabular}{lll}
\hline & Non-IORT $(n=21)$ & IORT $(n=20)$ \\
\hline Pre & $12(57 \%)$ & $19(95 \%)$ \\
Post & $14(66 \%)$ & $1(5 \%)$ \\
& & no prolonged proliferation \\
\hline
\end{tabular}

Number and percentage of samples where bASC (breast adipos stromal cells) were isolated

IORT intraoperative radiotherapy 
Fig. 1 Characterization of breast-derived adipose stromal cells (bASC). a Phase contrast photomicrographs in $\mathrm{p} 0$ of a pre and post IORT sample. The typical mesenchymal stromal cells(MSC)-like morphology is apparent in the pre intraoperative radiotherapy (IORT) sample. All cells attaching from the post IORT sample show a senescent phenotype: cells lose their fibroblastoid shape, become flat and huge with bundles of stress fibers. To allow for better comparison, contrast and brightness were slightly adjusted. b Exemplary figures of adipogeneic (ADA, Oil Red O stain) and osteogenic (ODA, von Kossa stain) differentiation results displaying the negative controls (top rows) and the adipogenic/ osteogenic-differentiated samples (bottom rows). c, d Flow cytometric assessment of MSC markers and e, f markers indicating contamination and other markers used to characterize putative subpopulations of MSC. Data are expressed as percent positive cells or mean fluorescence values MFI (median FL-A). No differences were seen; thus data are merged from all conditions. $M F I$ mean fluorescence intensity, $A D A$ adipogenic differntiation assay, $O D A$ osteogneic differentiation assay a Morphology at p0

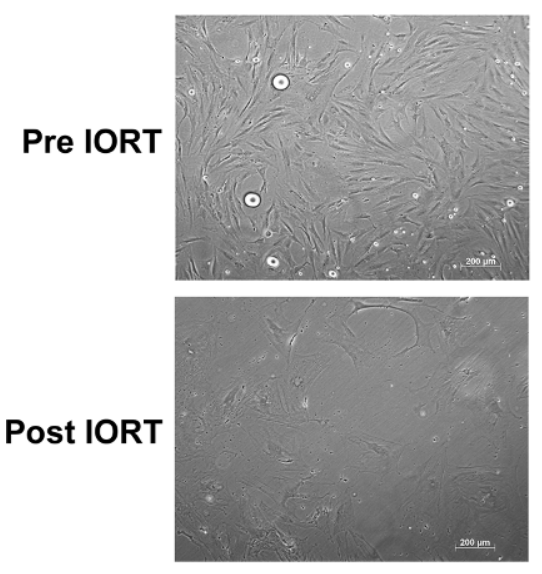

C

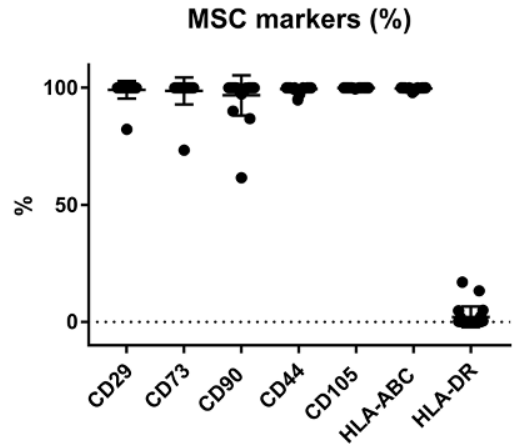

e contamination and putative markers $(\%)$

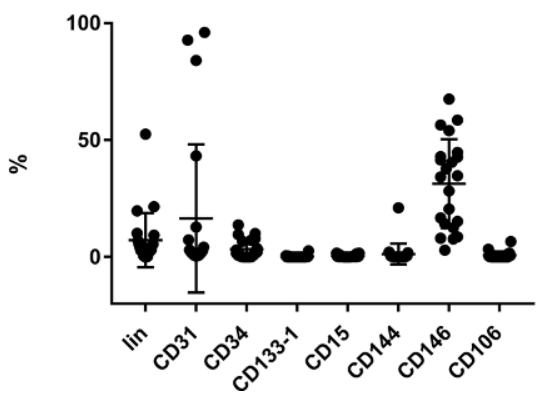

b non-IORT pre post

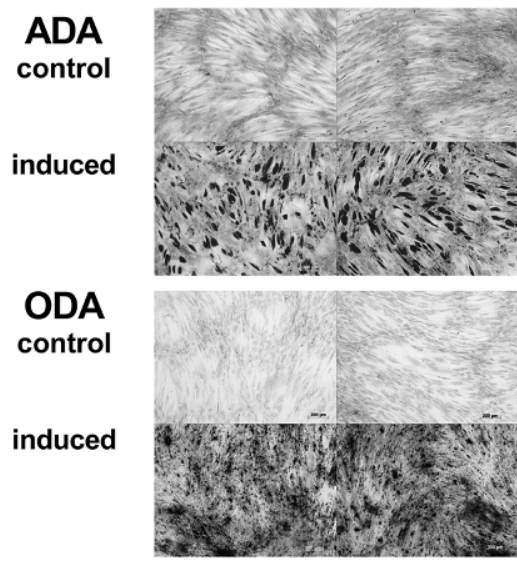

d MSC markers (MFI)

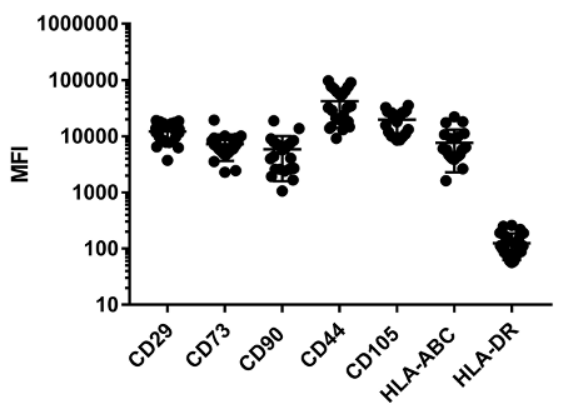

f

contamination and putative markers (MFI)

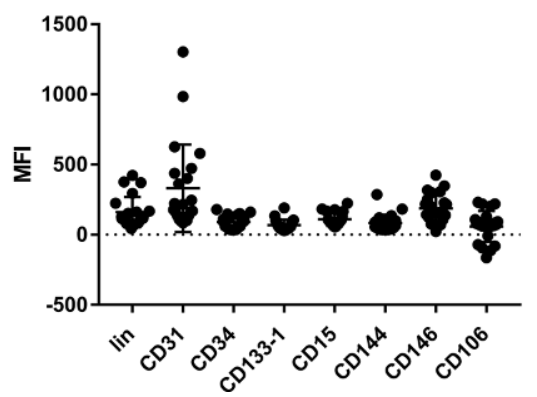

comparable with $0.45 \pm 0.37$ for pre non-IORT, $0.37 \pm 0.26$ post non-IORT and $0.56 \pm 0.44$ and $0.54 \pm 0.29 \mathrm{~g}$ for pre and post IORT, respectively. In all, $95 \%$ of the pre IORT samples and $57 \%$ and $66 \%$ of the pre and post nonIORT samples yielded MSC cultures, growing beyond passage 2 (Table 1). However, adherent cells were observed in only one of the 20 samples post IORT. These, however, stopped proliferation already after a few days, reminiscent of a highly senescent phenotype marked by numerous stress fibers (Fig. 1a; [7]). In all other cultures, bASC in early in culture grew out as colonies forming cell monolayers with a typical fibroblastoid phenotype (Fig. 1a). The proliferation potential of non-IORT bASC, both pre and post, was comparable to ASC isolated from subcutaneous fat [7]. bASC from pre IORT samples proliferated significantly slower (Table 2). bASC from two exemplary cultures could be cultured up to 6-7 passages until reaching replicative senescence, without an indication of excessive or prolonged proliferation indicative of malignant transformation or tumorigenic origin (not shown).

\section{Differentiation assay}

Differentiation assays and immunophenotyping were performed to verify the MSC-like nature of bASCs. Nearly $90 \%$ of the presamples underwent adipogenic differentia- 
Table 2 Growth curves

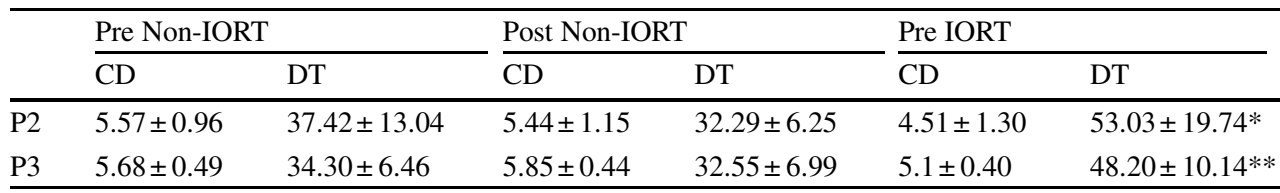

Cell doublings (CD) and doubling time (DT, in hours) are listed

IORT intraoperative radiotherapy

*DT p2: Post Non-IORT vs. pre-IORT $p=0.0355$

**DT p3: Pre and post Non-IORT to Pre-IORT $p=0.0225$ and $p=0.0119$, respectively

Table 3 Differentiation potential

\begin{tabular}{llll}
\hline & Pre Non-IORT & Post Non-IORT & Pre IORT \\
\hline Adipogenic & $88.89 \%$ & $54.55 \%$ & $87.5 \%$ \\
Osteogenic & $100 \%$ & $76.92 \%$ & $100 \%$ \\
\hline
\end{tabular}

Percentage of samples that underwent adipogenic or osteogenic differentiation upon inductive medium treatment

$I O R T$ intraoperative radiotherapy

tion, whereas from the post-non-IORT samples only 55\% showed adipogenic differentiation properties (Table 3 and Fig. 1b). All bASC presamples differentiated into the osteogenic lineage, whereas this proportion again was reduced to $77 \%$ in the post non-IORT samples (Table 3 and Fig. 1b).

\section{Immune phenotype}

The immune phenotype of bASC at p2 was highly typical for ASC and corresponded to the ISCT proposed positive and negative markers to distinguish MSCs from other cells $[12,13]$. As expected, no difference in the marker expression of bASC was observed comparing pre/post non-IORT and pre IORT samples. Thus, all data were merged. Furthermore, cells expressed/non-expressed the typical MSC markers (Fig. 1c, d) at comparable intensities in all but one sample. Negativity of HLA class II indicates a nonactivated state of isolated bASC. Markers to control for contamination (such as lineage for hematopoietic cells) were not detected in the majority (Fig. 1e, f). Surprisingly, few samples contained a high proportion of CD31-postive cells, indicating an endothelial trait despite their fibroblastoid morphology. Other endothelial markers such as CD144 (VE-Cadherin) and CD106 (VCAM), however, were not expressed. In p2, CD34 was not expressed, although ASC may express CD34 early after isolation [13]. The CD146+ subset has been suggested to represent a population transitional between adipose stromal cells and pericytes. In fact, percentage of expressing cells varied markedly. Mean fluorescence intensity on positive cells, however, was highly comparable.

\section{Discussion}

Our results indicate that IORT entirely abolishes the adhesion and proliferation potential of bASC, suggesting radiosensitivity of bASC, at least in situ. Since IORT is performed with $20 \mathrm{~Gy}$ prescribed to the applicator surface in a single session, supporting data that doses higher than 10 Gy radiosensitize MSC $[9,14]$. The morphology of the one post IORT sample, in which few cells attached and proliferated, resembled a senescent phenotype [7]. Alessio et al. described a senescent MSC phenotype induced by radiation ranging from $40 \mathrm{mGy}$ to $2 \mathrm{~Gy}$ in line with a loss of clonogenic capacity [15]. They showed that the few surviving clones, however, retained differentiation capacity fitting to previously published data suggesting a radioresistant subpopulation [14].

Despite the nearly high isolation success rate, pre IORT samples showed decelerated proliferation compared to nonIORT controls. Different subtypes or molecular phenotypes between the study and control cohort cannot explain these differences between pre IORT and pre/post non-IORT. The lower mean breast size in non-IORT women (exclusion criterion for IORT) and the likely different breast composition with a higher proportion of connective tissue, which was often observed in non-IORT samples, may explain the differing success rates. Although we tried to avoid thermocoagulation during biopsy collection, it is inevitable during breast-conserving surgery depending of the degree of bleeding. Nevertheless, within the groups there was no difference, so that we consider this effect as negligible.

Our data on osteogenic and adipogenic differentiation potential suggest that within the $30 \mathrm{~min}$ period after resecting the tumor and closing, the wound affects the differentiation - but not the outgrowth and proliferation-properties of bASC. Possibly the surgical stress induced (pro)inflammatory priming may has affected differentiation, previously shown to affect the balance between osteo- and adipogenic differentiation [14]. At the present stage, we cannot exclude that the extended stress caused by surgical tumor resection and IORT irradiation and subsequent biopsy affected bASC outgrowth rather than the IORT itself. We regard it important that the entire IORT procedure is effective in targeting the tumor niche. 
Growth, morphology, differentiation, and immune phenotypic characterization documented that bASC fulfilled criteria of MSCs. IORT ultimately abolished the outgrowth capacity of bASC, indicating bASC radiosensitivity in situ. These data support the notion that IORT exerts an ablative effect within the tumor bed, not only affecting potential residual tumor cells, but also the tumor bed. By this, the IORT concept of localized radiation targeting residual tumor cells in the tumor bed to reduce the risk of relapse appears to be validated with respect to tumor bed-derived bASC. Our data may aid in explaining the efficiency of IORT concerning local recurrences and overall survival as stated in the follow-up of the TARGIT A trial [16]. They may further argue towards a reduced risk of breast cancer metastasis [17].

It was beyond the scope of this study to assess the effect of radiation of expanded MSC $[9,11,14]$ and to investigate the potential impact of ionizing radiation on de novo recruitment of circulating MSC to the tumor bed as previously suggested [18, 19]. The question, if and how IORT may affect the recruitment of cells to the tumor bed and its local microenvironment, offers potential for future investigations.

\section{Conclusion}

After breast-conserving surgery, approximately $90 \%$ of local recurrences emerge in close proximity to the initial tumor bed, potentially reflecting a significant contribution of the tumor bed to relapse. The goal of intraoperative radiotherapy (IORT) is to target remaining tumor cells by a locally concentrated dose of radiation while preserving healthy tissue. Our results indicate that IORT, besides the proven effect on breast cancer cells, entirely abolishes the adhesion and proliferation potential of bASC, suggesting radiosensitivity of bASC at doses of $20 \mathrm{~Gy}$. This might add to reduce the risk of tumor relapse and metastases.

Funding Open Access funding provided by Projekt DEAL.

\section{Compliance with ethical guidelines}

Conflict of interest S. Uhlig, A. Wuhrer, S. Berlit, B. Tuschy, M. Sütterlin and K. Bieback declare that they have no competing interests.

Ethical standards Ethical approval: No. 2013-589N-MA, Mannheim Ethics committee II, University Medical Center Mannheim, Heidelberg University, Germany.

Open Access This article is licensed under a Creative Commons Attribution 4.0 International License, which permits use, sharing, adaptation, distribution and reproduction in any medium or format, as long as you give appropriate credit to the original author(s) and the source, provide a link to the Creative Commons licence, and indicate if changes were made. The images or other third party material in this article are included in the article's Creative Commons licence, unless indicated otherwise in a credit line to the material. If material is not included in the article's Creative Commons licence and your intended use is not permitted by statutory regulation or exceeds the permitted use, you will need to obtain permission directly from the copyright holder. To view a copy of this licence, visit http://creativecommons.org/licenses/by/4. $0 \%$.

\section{References}

1. Welzel G, Boch A, Sperk E, Hofmann F, Kraus-Tiefenbacher U, Gerhardt A, Suetterlin M, Wenz F (2013) Radiation-related quality of life parameters after targeted intraoperative radiotherapy versus whole breast radiotherapy in patients with breast cancer: results from the randomized phase III trial TARGIT-A. Radiat Oncol 8:9. https://doi.org/10.1186/1748-717X-8-9

2. Herskind C, Ma L, Liu Q, Zhang B, Schneider F, Veldwijk MR, Wenz F (2017) Biology of high single doses of IORT: RBE, 5R's, and other biological aspects. Radiat Oncol 12(1):24. https://doi.org/ 10.1186/s13014-016-0750-3

3. Demicheli R, Valagussa P, Bonadonna G (2001) Does surgery modify growth kinetics of breast cancer micrometastases? Br J Cancer 85(4):490-492. https://doi.org/10.1054/bjoc.2001.1969

4. Son B, Lee S, Youn H, Kim E, Kim W, Youn B (2017) The role of tumor microenvironment in therapeutic resistance. Oncotarget 8(3):3933-3945. https://doi.org/10.18632/oncotarget.13907

5. Piotrowski I, Kulcenty K, Murawa D, Suchorska W (2018) Surgical wound fluids from patients treated with intraoperative radiotherapy induce radiobiological response in breast cancer cells. Med Oncol 36(2):14. https://doi.org/10.1007/s12032-018-1243-z

6. Belletti B, Vaidya JS, D'Andrea S, Entschladen F, Roncadin M, Lovat F, Berton S, Perin T, Candiani E, Reccanello S, Veronesi A, Canzonieri V, Trovo MG, Zaenker KS, Colombatti A, Baldassarre G, Massarut S (2008) Targeted intraoperative radiotherapy impairs the stimulation of breast cancer cell proliferation and invasion caused by surgical wounding. Clin Cancer Res 14(5):1325-1332. https:// doi.org/10.1158/1078-0432.ccr-07-4453

7. Bieback K, Hecker A, Schlechter T, Hofmann I, Brousos N, Redmer T, Besser D, Kluter H, Muller AM, Becker M (2012) Replicative aging and differentiation potential of human adipose tissue-derived mesenchymal stromal cells expanded in pooled human or fetal bovine serum. Cytotherapy 14(5):570-583. https://doi.org/10.3109/ 14653249.2011.652809

8. Guo M, Dong Z, Qiao J, Yu C, Sun Q, Hu K, Liu G, Wei L, Yao B, Man Q, Sun X, Liu Z, Song Z, Yu C, Chen Y, Luo Q, Liu S, Ai HS (2014) Severe acute radiation syndrome: treatment of a lethally 60Co-source irradiated accident victim in China with HLA-mismatched peripheral blood stem cell transplantation and mesenchymal stem cells. J Radiat Res 55(2):205-209. https://doi. org/10.1093/jrr/rrt102

9. Nicolay NH, Lopez Perez R, Saffrich R, Huber PE (2015) Radio-resistant mesenchymal stem cells: mechanisms of resistance and potential implications for the clinic. Oncotarget 6(23):19366-19380. https://doi.org/10.18632/oncotarget.4358

10. Bartsch K, Al-Ali H, Reinhardt A, Franke C, Hudecek M, Kamprad M, Tschiedel S, Cross M, Niederwieser D, Gentilini C (2009) Mesenchymal stem cells remain host-derived independent of the source of the stem-cell graft and conditioning regimen used. Transplantation 87(2):217-221. https://doi.org/10.1097/TP. Ob013e3181938998

11. D'Andrea FP, Horsman MR, Kassem M, Overgaard J, Safwat A (2012) Tumourigenicity and radiation resistance of mesenchymal stem cells. Acta Oncol 51(5):669-679. https://doi.org/10.3109/ 0284186x.2011.636752 
12. Dominici M, Le Blanc K, Mueller I, Slaper-Cortenbach I, Marini F, Krause D, Deans R, Keating A, Prockop D, Horwitz E (2006) Minimal criteria for defining multipotent mesenchymal stromal cells. The international society for cellular therapy position statement. Cytotherapy 8(4):315-317. https://doi.org/10.1080/ 14653240600855905

13. Bourin P, Bunnell BA, Casteilla L, Dominici M, Katz AJ, March KL, Redl H, Rubin JP, Yoshimura K, Gimble JM (2013) Stromal cells from the adipose tissue-derived stromal vascular fraction and culture expanded adipose tissue-derived stromal/stem cells: a joint statement of the International Federation for Adipose Therapeutics and Science (IFATS) and the International Society for Cellular Therapy (ISCT). Cytotherapy 15(6):641-648. https://doi.org/10. 1016/j.jcyt.2013.02.006

14. Fekete N, Erle A, Amann EM, Furst D, Rojewski MT, Langonne A, Sensebe L, Schrezenmeier H, Schmidtke-Schrezenmeier G (2015) Effect of high-dose irradiation on human bonemarrow-derived mesenchymal stromal cells. Tissue Eng Part C Methods 21(2):112-122. https://doi.org/10.1089/ten.TEC.2013. 0766

15. Alessio N, Del Gaudio S, Capasso S, Di Bernardo G, Cappabianca S, Cipollaro M, Peluso G, Galderisi U (2015) Low dose radiation induced senescence of human mesenchymal stromal cells and impaired the autophagy process. Oncotarget 6(10):8155-8166. https://doi.org/10.18632/oncotarget.2692
16. Vaidya JS, Wenz F, Bulsara M, Tobias JS, Joseph DJ, Keshtgar M, Flyger HL, Massarut S, Alvarado M, Saunders C, Eiermann W, Metaxas M, Sperk E, Sutterlin M, Brown D, Esserman L, Roncadin M, Thompson A, Dewar JA, Holtveg HM, Pigorsch S, Falzon M, Harris E, Matthews A, Brew-Graves C, Potyka I, Corica T, Williams NR, Baum M (2014) Risk-adapted targeted intraoperative radiotherapy versus whole-breast radiotherapy for breast cancer: 5-year results for local control and overall survival from the TARGIT-A randomised trial. Lancet 383(9917):603-613. https:// doi.org/10.1016/S0140-6736(13)61950-9

17. Karnoub AE, Dash AB, Vo AP, Sullivan A, Brooks MW, Bell GW, Richardson AL, Polyak K, Tubo R, Weinberg RA (2007) Mesenchymal stem cells within tumour stroma promote breast cancer metastasis. Nature 449(7162):U557-U554. https://doi.org/10.1038/ nature 06188

18. Klopp AH, Spaeth EL, Dembinski JL, Woodward WA, Munshi A, Meyn RE, Cox JD, Andreeff M, Marini FC (2007) Tumor irradiation increases the recruitment of circulating mesenchymal stem cells into the tumor microenvironment. Cancer Res 67(24):11687-11695. https://doi.org/10.1158/0008-5472.Can-071406

19. Zielske SP, Livant DL, Lawrence TS (2009) Radiation increases invasion of gene-modified mesenchymal stem cells into tumors. Int J Radiat Oncol Biol Phys 75(3):843-853. https://doi.org/10.1016/j. ijrobp.2008.06.1953 